\title{
Eficacia analgésica del tratamiento invasivo miofascial (punción seca) en fibromialgia
}

\author{
JUAN VICENTE MAMPEL \\ Juanvicentemampel@yahoo.es \\ FRANCISCO ROS BERNAL \\ fros@uji.es
}

\section{Resumen}

Introducción. La fibromialgia (FM) es una entidad clínica caracterizada por un dolor crónico generalizado. La sensibilización central del sistema nervioso se considera la explicación más plausible al dolor crónico generalizado que afecta a las personas con FM. La nueva neurobiología del dolor establece que los puntos gatillo miofasciales puede ser fuente periférica capaz de originar el dolor. El tratamiento de los PGM puede disminuir el dolor de los pacientes con FM. Metodología. Se realizó un ensayo clínico aleatorizado con 120 participantes, se diseñaron 3 grupos con el objetivo de comprobar la eficacia de la intervención. Resultados. Los resultados obtenidos determinan diferencias significativas entre el grupo Tratamiento y grupo Placebo $(p=0,01)$. Conclusiones. Los pacientes sometidos a la técnica de punción seca mostraron una disminución de la hiperalgesia local a las 24 horas de la intervención.

Palabras clave: Dolor miofascial, fibromialgia, punción seca, dolor crónico.

\begin{abstract}
Introduction. Fibromyalgia (FM) is a clinical entity characterized by widespread chronic pain. Central sensitization of the nervous system is considered the most plausible explanation for the widespread chronic pain that affects people with FM. The new neurobiology of pain states that myofascial trigger points can be a peripheral source capable of causing pain. Treatment of MTrPs may decrease the pain of patients with FM. Methodology. We designed a randomized clinical trial with 120 participants divided into 3 groups with the objective of checking the effectiveness of the intervention. Results. The results obtained determined significant differences between the treatment and the Placebo group $(p=0.006)$. Conclusions. Patients undergoing the dry needling technique showed a decrease in local hyperalgesia 24 hours after surgery.
\end{abstract}

Key Words: Myofascial pain, fibromyalgia, dry needling, chronic pain. 


\section{Introducción}

La fibromialgia (FM) es una entidad clínica caracterizada por un dolor crónico generalizado. Su consideración como enfermedad permite abarcar todo el conjunto de componentes comórbidos que acompañan al dolor, entre los que destacan la fatiga, la disfunción cognitiva y los trastornos del sueño (Chinn, Caldwell y Gritsenko, 2016). La FM es una enfermedad que en la actualidad afecta al $2,4 \%$ de la población española generando un gasto sanitario en el territorio español de aproximadamente 11.000 .000 euros al año.

La sensibilización central del sistema nervioso se considera la explicación más plausible al dolor crónico generalizado que afecta a las personas con FM. A pesar de la etiología incierta establecida en la actualidad la evidencia sugiere que la sensibilización central, aunque no es la única causa (Yunus, 2007), origina un estado aumentado de la sensibilidad, que produce un dolor generalizado y difuso en el sujeto con FM (Butler y Moseley, 2010).

La etiopatología anteriormente descrita de la FM se establece a partir del cambio en cuanto al entendimiento del dolor. La concepción del dolor ha estado estrechamente ligada a la teoría darwiniana de la evolución y, recientemente, de un modelo biomédico simplista y obsoleto se ha cambiado a un modelo biopsicosocial donde no solo el componente biológico sino también el psicológico y social forman parte de cualquier proceso álgico.

Esta nueva perspectiva del dolor atribuye al dolor miofascial y concretamente a los puntos gatillos miofasciales (PGM) como estructuras periféricas capaces de mantener la nocicepción en un cuadro de sensibilización central. De esta manera, la información nociceptiva procedente de estas estructuras constituiría un factor predisponente implicado en la neurobiología del dolor crónico.

El dolor miofascial,encuadrado dentro de las mialgias, es una alteración no inflamatoria originada en el sistema músculo-esquelético y con la presencia de PGM, nódulos hiperirritables, situados en las fibras del músculo. Es un dolor de tipo músculo-esquelético, agudo o crónico que a menudo es infradiagnosticado.

Una vez se ha establecido que existe incremento de la sensibilización central originado por una activación del PGM es recomendable reducir la hiperalgesia local en un enfermo de FM a través del tratamiento de los PGM. Al tratar el componente periférico se obtiene una mejora, tanto del dolor local como generalizado, disminuyendo y reduciendo la sensibilización periférica. El objetivo principal del presente estudio fue valorar los efectos de la punción seca en la hiperalgesia local de pacientes con FM.

\section{Material y método}

Realizamos un ensayo clínico aleatorizado con tres grupos de pacientes diagnosticadas con FM: i) un grupo experimental, ii) un grupo control placebo y iii) un grupo control sin intervención. El grupo experimental (Tratamiento) lo constituyeron los sujetos a los que se les realizó la técnica de punción seca. A las pacientes del grupo control placebo (Placebo), se les simuló la punción. Por último, al grupo control sin intervención (No intervención), no se les realizó nada.

Se utilizó la guía CONSORT, con las recomendaciones STRICTA (MacPherson y cols., 2010) para establecer las directrices del proceso experimental y evitar cualquier sesgo durante todo el proceso. Todo el protocolo del estudio fue aprobado por la Comisión Deontológica de la Universitat Jaume I (Castellón), de acuerdo con la Declaración de Helsinki de 1975, revisada en 1983. 
Se empleó la técnica de enmascaramiento (doble ciego). La asignación al grupo se determinó a través del programa EPIDAT 3.1 (Xunta de Galicia). Los criterios de inclusión para participar en el estudio fueron: i) ser mujer, ii) haber recibido un diagnóstico de FM por un reumatólogo, iii) tener entre 35 y 80 años, iv) ser capaz de entender el español y v) haber firmado el consentimiento informado por decisión propia. Por otro lado, los criterios de exclusión fueron: i) sufrir belonefobia, ii) presentar un linfedema en la zona a tratar, ya que éste aumenta el riesgo de infección por la punción, iii) estar en estado de gestación o con la posibilidad de estarlo, iv) personas sometidas con anterioridad a técnicas de punción seca o v) diagnosticadas de enfermedades neoplásicas.

En todas las participantes pertenecientes a los grupos tratamiento y placebo se localizaron los PGM del músculo infraespinoso del brazo dominante. En el grupo tratamiento se realizó la técnica de entradas y salidas rápidas de Hong (Hong, 1994) una vez obtenida la primera reacción de espasmo local (REL) la aguja se movió unos 2-3 $\mathrm{mm}$ en vertical de forma rápida y realizándose aproximadamente 25 inserciones sin salir de la piel, con una frecuencia aproximada de $1 \mathrm{~Hz}$ durante 25 a 30 segundos (Arias-Buría y cols., 2015). Los pacientes del grupo Placebo fueron sometidos a una intervención que simulaba la punción empleando una aguja placebo de mango retraíble (Streitberger y Kleinhenz, 1998). El grupo de No intervención se diseñó para dilucidar si la intervención era una técnica de enmascaramiento de la punción seca. A las pacientes no se les localizaron los PGM, ni se les realizó punción seca, ni la simulación de la misma.

La algometría por presión fue el método utilizado para valorar la eficacia de la punción seca. Para ello empleamos un instrumento denominado algómetro con un manómetro adherido a una punta de goma cilíndrica. La fiabilidad de la algometría por presión es relativamente alta, con coeficientes de 0,9 y 0,95 (Chesterton, Sim, Wright y Foster, 2007). Se aplicó la medición algométrica en el epicóndilo lateral del brazo ipsilateral que corresponde al esclerotoma de la raíz C5-C6, cuyo miotoma se asocia al lugar de la intervención (músculo infraespinoso). Se realizaron mediciones antes del tratamiento/placebo, 5 minutos después y a las 24 horas post tratamiento.

La variable algometría local se describió empleando una medida de tendencia central (media), de dispersión (desviación típica), coeficiente de Curtosis y coeficiente de Asimetría. Las medias se compararon mediante el test exacto de ANOVA (corrección con Bartlett's test) y si la diferencia era significativa, se realizaron consecuentes contrastes post hoc (comparaciones múltiples). Se aceptó un nivel de riesgo alfa del 0,05. Todos los análisis estadísticos se realizaron empleando el programa Statistical Package for the Social Sciences (SPSS) versión 23.0 IBM® para Windows XP.

\section{Resultados}

De los 250 sujetos reclutados en el estudio se excluyeron 130 participantes. Los principales motivos por los cuales se descartaron fueron: i) el no cumplimiento de los criterios de inclusión (19,9\%), ii) negativa a participar a pesar de cumplir los criterios de inclusión $(28,4 \%)$ y iii) otros motivos (4\%). Con los 120 participantes aptos para el estudio se conformaron los tres grupos y una vez iniciado el estudio de campo, tras la firma del consentimiento informado, finalizaron el estudio de manera completa 34 sujetos del grupo tratamiento, 30 sujetos del grupo placebo y 32 sujetos del grupo no intervención. De esta manera la tasa de abandono del estudio fue de un $20 \%$.

Tras la realización del análisis inferencial de las variables antropométricas de los sujetos pertenecientes al estudio se desprende que la aleatorización fue existosa (ver Tabla1). 
Tabla 1

Comparación de las variables antropométricas entre grupos. Se realizó la prueba de ANOVA para determinar si existían diferencias significativas en los grupos para las variables edad, peso, talla e IMC

\begin{tabular}{ccc}
\hline & ANOVA (Entre grupos) & \\
\hline Variable & $\mathrm{F}$ & p-valor \\
\hline Edad & 1,19 & 0,31 \\
Peso & 0,69 & 0,50 \\
Talla & 2,29 & 0,12 \\
IMC & 0,08 & 0,92 \\
\hline
\end{tabular}

F: estimación varianza poblacional; No se observaron diferencias significativas en ninguna de las variables analizadas $p>0,050$.

El análisis descriptivo de la algometría local desprendió los valores mostrados a continuación (ver Tabla 2).

Tabla 2

Estadísticos descriptivos de la algometría local. Representación de la media, desviación estándar, Asimetría y Curtosis

\begin{tabular}{|c|c|c|c|c|c|}
\hline \multicolumn{6}{|c|}{ Algometría local (pretratamiento) } \\
\hline & $\mathrm{N}$ & Media & $\begin{array}{l}\text { Desviación } \\
\text { estándar }\end{array}$ & Asimetría & Curtosis \\
\hline Tratamiento & 34 & 25,57 & 7,54 & 0,49 & $-0,49$ \\
\hline Placebo & 30 & 22,68 & 8,48 & 0,35 & $-0,62$ \\
\hline No intervención & 32 & 27,00 & 8,82 & 0,16 & $-0,70$ \\
\hline \multicolumn{6}{|c|}{ Algometría local (postratamiento) } \\
\hline & $\mathrm{N}$ & Media & $\begin{array}{l}\text { Desviación } \\
\text { estándar }\end{array}$ & Asimetría & Curtosis \\
\hline Tratamiento & 34 & 26,63 & 7,95 & 0,12 & 0,11 \\
\hline Placebo & 30 & 22,23 & 7,51 & 0,59 & 0,57 \\
\hline No intervención & 32 & 25,30 & 8,85 & $-0,15$ & $-1,24$ \\
\hline \multicolumn{6}{|c|}{ Algometría local (post24 horas tratamiento) } \\
\hline Tratamiento & 34 & 26,77 & 7,94 & 0,54 & $-0,43$ \\
\hline Placebo & 30 & 20,90 & 6,69 & 0,77 & 1,44 \\
\hline No intervención & 32 & 24,31 & 7,40 & 0,21 & $-0,51$ \\
\hline
\end{tabular}

Pretratamiento: medición antes intervención: postratamiento: medición tomada 5 minutos después y post-24 horas tratamiento: medición tomada a las 24 horas de la intervención. 
Al realizar el análisis estadístico a través de la ANOVA de un factor no se demostró la existencia de diferencias significativas entre grupos dentro del valor algometría local post intervención según el tratamiento recibido $(p=0,10)$. En cambio, sí existen diferencias significativas entre los grupos al analizar la algometría local 24 horas post intervención $(p=0,01)$ (ver Tabla 3 y Figura 2).

Tabla 3

Análisis ANOVA. Comparación de variables de la VFC entre grupos. Comparación variable algometría local postratamiento y algometría local post 24 horas entre grupos. Se realizó la prueba ANOVA para comprobar si existían diferencias significativas entre los grupos

\begin{tabular}{ccc}
\hline & ANOVA (Entre grupos) \\
\hline Variable & $\mathrm{F}$ & p-valor \\
\hline Algometría local Post & 2,42 & 0,10 \\
Algometría local post 24 horas & 5,04 & $\underline{0,01}$ \\
\hline
\end{tabular}

F: estimación varianza poblacional. En este caso, se observaron dichas diferencias en la algometría local post 24 horas p>0,01.

Una vez comprobado la significancia obtenida tras el análisis ANOVA, se procedió a realizar la prueba post hoc utilizando la HDS de Tukey al comprobar la homogeneidad de varianzas. Los resultados obtenidos determinan diferencias significativas entre el grupo Tratamiento y grupo Placebo $(p=0,01)$. Sin embargo, no existían diferencias significativas entre el grupo Placebo o el grupo tratado frente al no intervenido $(p=0,17)$ (ver Tabla 4). Aun así, las tendencias de las medias determinan un efecto mayor sobre los valores de la algometría local a las 24 horas de la intervención en el grupo tratado con punción seca (ver Figura 2).

Tabla 4

Análisis post hoc. Comparación entre grupos algometría local post 24 horas

\begin{tabular}{ccc}
\hline \multicolumn{3}{c}{ Comparaciones múltiples (HSD Tukey) } \\
\hline Grupos & Algometría Local Post & Algometría Local Post 24h \\
\hline Tto/placebo & $p=0,08$ & $p=\underline{0,01}$ \\
Tto/no int & $p=0,78$ & $p=0,37$ \\
Placebo/no int & $p=0,30$ & $p=0,17$ \\
\hline
\end{tabular}

Tto: Grupo Tratamiento; Placebo: Grupo intervención simulada; no int: Grupo No intervención. En esta variable se muestra una diferencia entre el grupo Tratamiento y el grupo Placebo $p=0,01$. Además, en esta misma variable no se muestra diferencia entre el grupo Placebo y no intervención $p=0,17$. 


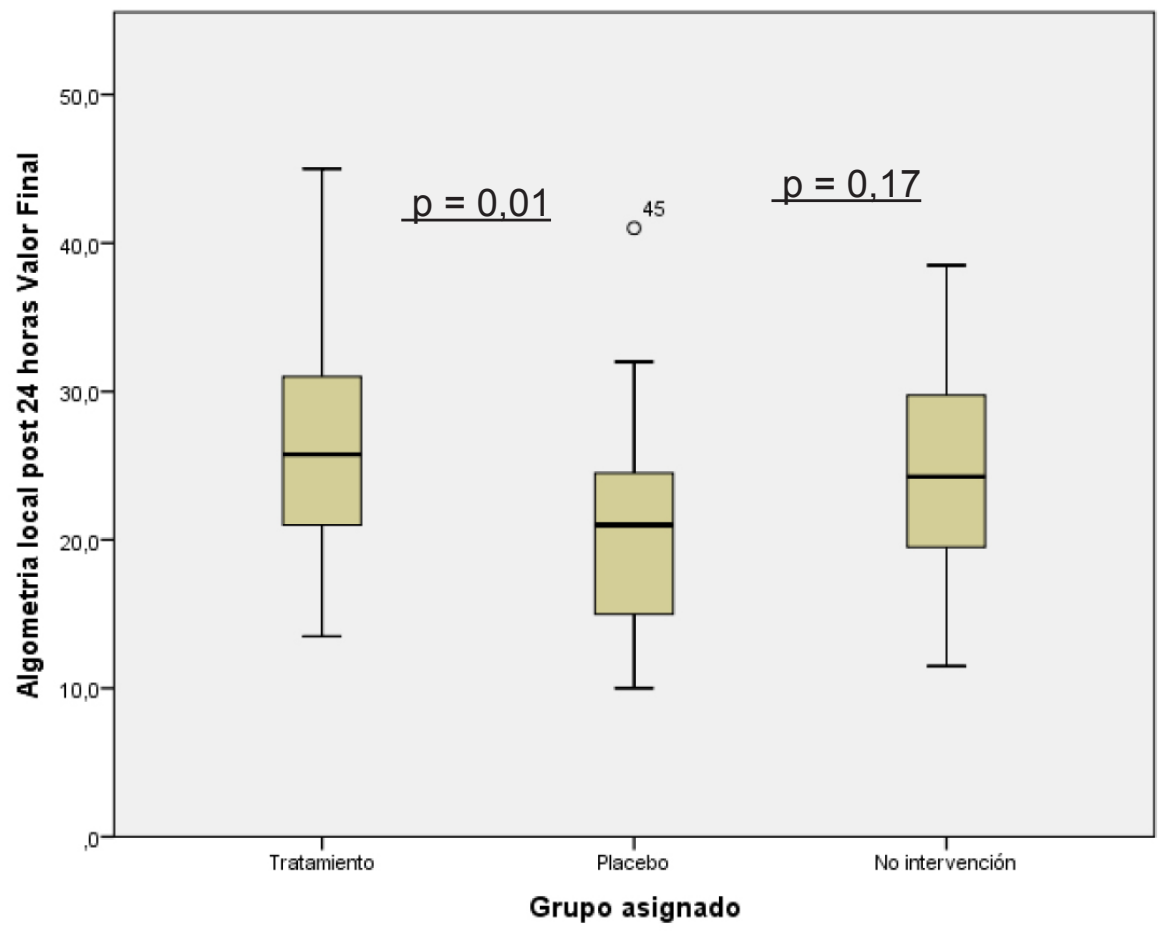

Figura 2. Representación gráfica de la media de los tres grupos algometría local post 24 horas. El diagrama de cajas muestra las diferencias significativas entre las medias de los distintos grupos de intervención

\section{Discusión y conclusiones}

Con los resultados obtenidos y representados con anterioridad, a primera vista se podría dilucidar una disminución de los efectos de los valores de la hiperalgesia local tras la punción seca en en personas con fibromialgia. Pero, ¿este tratamiento sería capaz de producir analgesia en este tipo de pacientes?

Los componentes de cada segmento espinal se denominan dermatoma, miotoma y esclerotoma. La importancia de los PGM en la fisiopatología de la sensibilización periférica es clave a la hora de determinar el daño de los tejidos a través de la liberación de sustancias algógenas. Todo el diseño experimental está basado en la sensibilización espinal segmentaria, el segmento espinal utilizado para el estudio fue la raíz nerviosa C5-C6 cuyo miotoma corresponde al musculo infraespinoso, y el esclerotoma al epicóndilo lateral (Fischer, 2002). Los PGM del músculo infraespinoso (raíz C6) se consideran focos irritativos capaces de generar un alto flujo de estímulos nociceptivos hacia el ganglio dorsal sensitivo de la médula espinal ( Bonica, 1990) manteniendo un cuadro de sensibilización central.

La técnica de punción fue elegida con el objetivo de conseguir la REL puesto que se ha comprobado un aumento de la eficacia de la técnica al provocar dicho efecto fisiológico (Rha y cols., 2011). En ningún momento de la intervención el paciente pudo percibir visualmente cómo fueron introducidas las agujas al estar colocado en decúbito lateral y realizar la intervención en el vientre muscular del músculo infraespinoso, siguiendo el procedimiento realizado en otros estudios de punción seca (Huguenin y cols., 2005). En esta línea, estudios previos han constatado que el hecho de ver la aguja simuladora por parte del paciente puede originar un 
fracaso de la técnica de enmascaramiento para el grupo placebo (Wong, Leung y Zhang, 2015).

En relación con la técnica de enmascaramiento algunos estudios no consideran que la técnica de punción simulada pueda ser útil como método de enmascaramiento ya que la relación con el agente inactivo asociado al componente psicológico puede generar efectos semejantes al tratamiento. La presión ejercida con la punta roma de la aguja y la presión durante la palpación del PGM activan la estimulación neurosensorial y las vías aferentes que inervan la piel, llegando hasta los centros superiores, por tanto esta estimulación sensorial podría tener un posible efecto terapéutico (Lund y Lundeberg, 2006; Lund, Näslund y Lundeberg, 2009; Lundeberg, Lund, Näslund y Thomas, 2008). De igual manera, en estudios de acupuntura se considera la técnica con aguja de punción simulada una herramienta de enmascaramiento útil ante el grupo de intervención (Tough, White, Richards, Lord y Campbell, 2009).

Aunque no se han encontrado estudios que analizaran el uso y los posibles efectos (psicológicos y fisiológicos) de la técnica de punción simulada, como método de enmascaramiento de la punción seca, esta herramienta se ha empleado para cegar los ensayos clínicos que comprueban la eficacia del tratamiento invasivo miofascial (Dıraçoğlu, Vural, Karan y Aksoy, 2012; Tekin y cols., 2013). Por este motivo, en el presente estudio, se ha añadido un tercer grupo, el grupo No tratamiento, al que no se le ha introducido ninguna aguja, ya fuese experimental o sham. Además, en este último grupo no se realizó la localización a través de la palpación de los PGM. De esta forma se pudo dilucidar si la herramienta de punción simulada era útil para la intervención como técnica de enmascaramiento en los estudios de punción seca.

El dolor es el síntoma distintivo de la FM y, tal y como se estableció en el marco teórico, no depende del grado de daño o inflamación en los tejidos periféricos. La alteración del procesamiento sensorial que caracteriza a estos individuos crea una situación final de anormalidad local, aumentando la sensibilidad de los pacientes con FM (Cassisi y cols., 2014). Por ello, se considera que el umbral de dolor a la presión, medida que se ha tomado con un algómetro, es clave en la evaluación clínica de los sujetos con FM (Gómez-Perretta, Triñanes, GonzálezVillar y Carrillo-de-la-Peña, 2016).

Con la algometría local se cuantificó de manera objetiva y numérica el grado de hiperalgesia mecánica de los sujetos que participaron en el estudio. Se valoró si la amplificación dolorosa, la disminución del umbral de dolor a la presión (Jespersen y cols., 2007) característica de estos pacientes, se modificaba tras el tratamiento con punción seca. Staud y cols publicaron un estudio similar al de la presente tesis introduciendo infiltraciones de un anestésico local (lidocaína) en zonas frecuentes de dolor referidas por pacientes con FM. Sus resultados fueron concluyentes y demostraron la importancia del componente periférico para el mantenimiento de la hiperalgesia mecánica y térmica en FM. A pesar de que sus resultados fueron positivos y consiguieron cambios en cuanto a la clínica del dolor, determinaron que un alto porcentaje de la reducción del mismo se debía a interferencias tales como la analgesia producida por el efecto placebo y la analgesia producida por la propia inserción de la aguja dependiente de algún modo de la profundidad a la cual se insertaba (Staud, Weyl, Bartley, Price y Robinson, 2014).

En cuanto a los resultados que se obtuvieron respecto a la algometría local se mostró que la técnica de punción seca poseía efecto analgésico a las 24 horas de la intervención. El efecto analgésico fue mayor en el grupo Tratamiento y la diferencia fue estadísticamente significativa respecto al grupo Placebo. En cambio, no hubo diferencias entre el grupo Placebo y No intervención, determinando así una ausencia de la analgesia placebo producida por las interferencias de las creencias y pensamientos derivados de ser sometidos a una técnica de punción. Estos resultados positivos en cuanto a la efectividad del enmascaramiento con la técnica que simula la punción contrastan con resultados obtenidos en otros estudios de acu- 
puntura, donde no se encontraron diferencias entre el grupo Tratamiento y la técnica placebo utilizada (Deare y cols., 2013). La aplicación de la técnica en el PGM localizado a través de la palpación y confirmada su localización a través de la REL puede ser responsable del éxito actual del proceso de enmascaramiento. Así pues, la técnica utilizada para simular la punción es útil para introducirla en estudios con punción seca.

Hay que señalar que en el grupo Tratamiento fue en el único donde el umbral de dolor a la presión mostró un aumento en la comparación antes/después y antes/a las 24 horas. En los otros dos grupos este umbral descendió después de la intervención. Por lo tanto, se concluye que los pacientes sometidos a la técnica de punción seca mostraron una disminución de la hiperalgesia local a las 24 horas de la intervención en relación al grupo Placebo y al No intervención. Este efecto no se encontró en la valoración realizada inmediatamente después del tratamiento. La importancia del presente estudio reside en la disminución de la hiperalgesia en los pacientes con fibromialgia después de realizarles el tratamiento con punción seca. Es decir, el tratamiento de componente periférico, concretamente, de los PGM a través de la técnica elegida ha demostrado un efecto neuromodulador del dolor en este cuadro de sensibilización central.

\section{Referencias bibliográficas}

Arias-Buría, J. L., Valero-Alcaide, R., Cleland, J. A., Salom-Moreno, J., Ortega-Santiago, R., Atín-Arratibel, M. A., y Fernández-de-las-Peñas, C. (2015). Inclusion of trigger point dry needling in a multimodal physical therapy program for postoperative shoulder pain: a randomized clinical trial. Journal of Manipulative and Physiological Therapeutics, 38(3), 179-187. https://doi.org/10.1016/j.jmpt.2014.11.007

Butler, D. S. y Moseley, G. L. (2010). Explicando el Dolor. Noigroup Publications.

Cassisi, G., Sarzi-Puttini, P., Casale, R., Cazzola, M., Boccassini, L., Atzeni, F., y Stisi, S. (2014). Pain in fibromyalgia and related conditions. Reumatismo, 66(1), 72-86.

Chesterton, L. S., Sim, J., Wright, C. C. y Foster, N. E. (2007). Interrater reliability of algometry in measuring pressure pain thresholds in healthy humans, using multiple raters. The Clinical Journal of Pain, 23(9), 760-766. https://doi.org/10.1097/AJP.0b013e318154b6ae

Chinn, S., Caldwell, W. y Gritsenko, K. (2016). Fibromyalgia Pathogenesis and Treatment Options Update. Current Pain and Headache Reports, 20(4), 25. https://doi.org/10.1007/ s11916-016-0556-X

Deare, J. C., Zheng, Z., Xue, C. C. L., Liu, J. P., Shang, J., Scott, S. W., y Littlejohn, G. (2013). Acupuncture for treating fibromyalgia. The Cochrane Database of Systematic Reviews, (5), CD007070. https://doi.org/10.1002/14651858.CD007070.pub2

Dıraçoğlu, D., Vural, M., Karan, A., y Aksoy, C. (2012). Effectiveness of dry needling for the treatment of temporomandibular myofascial pain: a double-blind, randomized, placebo controlled study. Journal of Back and Musculoskeletal Rehabilitation, 25(4), 285-290. https://doi.org/10.3233/BMR-2012-0338

Fischer, A.A. (2002). Functional Diagnosis of musculoskeletal pain and evaluation of treatment results by quantitative and objetive techniques. In Myofascial Pain and Fibromyalgia. Trigger Point Management (2nd ed., pp. 145-173). Rachlin.

Gómez-Perretta, C., Triñanes, Y., González-Villar, A. J., y Carrillo-de-la-Peña, M. T. (2016). Evaluation of the accuracy of several symptoms and domains in distinguishing patients diagnosed with fibromyalgia from healthy controls. Clinical and Experimental Rheumatology, 34(2 Suppl 96), S14-25. 
Hong, C. Z. (1994). Lidocaine injection versus dry needling to myofascial trigger point. The importance of the local twitch response. American Journal of Physical Medicine y Rehabilitation / Association of Academic Physiatrists, 73(4), 256-263.

Huguenin, L., Brukner, P. D., McCrory, P., Smith, P., Wajswelner, H., y Bennell, K. (2005). Effect of dry needling of gluteal muscles on straight leg raise: a randomised, placebo controlled, double blind trial. British Journal of Sports Medicine, 39(2), 84-90. https://doi.org/10.1136/ bjsm.2003.009431

Jespersen, A., Dreyer, L., Kendall, S., Graven-Nielsen, T., Arendt-Nielsen, L., Bliddal, H., y Danneskiold-Samsoe, B. (2007). Computerized cuff pressure algometry: A new method to assess deep-tissue hypersensitivity in fibromyalgia. Pain, 131(1-2), 57-62. https://doi. org/10.1016/j.pain.2006.12.012

Bonica, J.J. (1990). The management of pain (2nd ed.). Philadelphia: Lea y Febiger.

Lund, I., y Lundeberg, T. (2006). Are minimal, superficial or sham acupuncture procedures acceptable as inert placebo controls? Acupuncture in Medicine: Journal of the British Medical Acupuncture Society, 24(1), 13-15.

Lund, I., Näslund, J., y Lundeberg, T. (2009). Minimal acupuncture is not a valid placebo control in randomised controlled trials of acupuncture: a physiologist's perspective. Chinese Medicine, 4, 1. https://doi.org/10.1186/1749-8546-4-1

Lundeberg, T., Lund, I., Näslund, J., y Thomas, M. (2008). The Emperors sham - wrong assumption that sham needling is sham. Acupuncture in Medicine: Journal of the British Medical Acupuncture Society, 26(4), 239-242.

MacPherson, H., Altman, D. G., Hammerschlag, R., Youping, L., Taixiang, W., White, A., ... on behalf of the STRICTA Revision Group. (2010). Revised STandards for Reporting Interventions in Clinical Trials of Acupuncture (STRICTA): Extending the CONSORT Statement. Journal of Evidence-Based Medicine, 3(3), 140-155. https://doi. org/10.1111/j.1756-5391.2010.01086.x

Rha, D., Shin, J. C., Kim, Y.-K., Jung, J. H., Kim, Y. U., y Lee, S. C. (2011). Detecting local twitch responses of myofascial trigger points in the lower-back muscles using ultrasonography. Archives of Physical Medicine and Rehabilitation, 92(10), 1576-1580.e1. https:// doi.org/10.1016/j.apmr.2011.05.005

Staud, R., Weyl, E. E., Bartley, E., Price, D. D., y Robinson, M. E. (2014). Analgesic and antihyperalgesic effects of muscle injections with lidocaine or saline in patients with fibromyalgia syndrome. European Journal of Pain (London, England), 18(6), 803-812. https:// doi.org/10.1002/j.1532-2149.2013.00422.x

Streitberger, K., y Kleinhenz, J. (1998). Introducing a placebo needle into acupuncture research. Lancet (London, England), 352(9125), 364-365. https://doi.org/10.1016/S01406736(97)10471-8

Tekin, L., Akarsu, S., Durmuş, O., Cakar, E., Dinçer, U., y Kıralp, M. Z. (2013). The effect of dry needling in the treatment of myofascial pain syndrome: a randomized double-blinded placebo-controlled trial. Clinical Rheumatology, 32(3), 309-315. https://doi.org/10.1007/ s10067-012-2112-3

Tough, E. A., White, A. R., Richards, S. H., Lord, B., y Campbell, J. L. (2009). Developing and validating a sham acupuncture needle. Acupuncture in Medicine: Journal of the British Medical Acupuncture Society, 27(3), 118-122. https://doi.org/10.1136/aim.2009.000737

Wong, E. L.-Y., Leung, P.-C., y Zhang, L. (2015). Placebo acupuncture in an acupuncture clinical trial. How good is the blinding effect? Journal of Acupuncture and Meridian Studies, 8(1), 40-43. https://doi.org/10.1016/j.jams.2014.10.010 
Yunus, M. B. (2007). Role of central sensitization in symptoms beyond muscle pain, and the evaluation of a patient with widespread pain. Best Practice y Research. Clinical Rheumatology, 21(3), 481-497. https://doi.org/10.1016/j.berh.2007.03.006 